\title{
CLDN6 promotes tumor progression through the YAP1-snail1 axis in gastric cancer
}

\author{
Site Yu (1)', Yeqian Zhang ${ }^{1}$, Qing Li' ${ }^{2}$ Zizhen Zhang ${ }^{1}$, Gang Zhao ${ }^{1}{ }^{1}$ and Jia Xu'
}

\begin{abstract}
Claudin6 (CLDN6), a member of the tight junction family, is a molecule involved in intercellular adhesion, acting as a physical barrier that prevents solutes and water from freely passing through the extracellular space. CLDN6 has important biological functions, and its abnormal expression is associated with Hepatitis $\mathrm{C}$ infection. However, there is limited research regarding its role in gastric cancer. In this study, we found that the expression of CLDN6 mRNA and protein was upregulated in gastric cancer cell lines and tissues, which indicated poor prognosis. Both in vitro and in vivo experiments showed that abnormal CLDN6 expression was associated with enhanced proliferation and invasion abilities of gastric cancer. CLDN6 reduced the phosphorylation of LATS1/2 and YAP1 by interacting with LATS1/2 in the Hippo signaling pathway. Thus, CLDN6 affected the entry of YAP1 into the nucleus, causing changes in downstream target genes. Moreover, YAP1 interacted with snail1 to affect the process of EMT and enhanced the invasive ability of GC cells. Collectively, CLDN6 promoted the proliferation and invasive ability of gastric cancer by affecting YAP1 and YAP1-snail1 axis.
\end{abstract}

\section{Introduction}

Gastric cancer is one of the five major malignant tumors that seriously endanger human health ${ }^{1}$. The incidence of gastric cancer is higher in East Asian countries, especially in China, Japan, and South Korea ${ }^{2}$. In China, gastric cancer ranks second and third in annual morbidity and mortality, respectively ${ }^{3}$. While the rate of diagnosis of early gastric cancer has improved with the advancement of medical technology, the overall rate of diagnosis remains poor ${ }^{4}$. Most patients with gastric cancer are in the middle and late stage when diagnosed, and the malignant degree of these two stages is high, often causing distant visceral metastasis through blood and lymphatic drainage, and other mechanisms ${ }^{5}$. Studies have shown

\footnotetext{
Correspondence: Gang Zhao (zhaogang74313@aliyun.com) or

Jia Xu (xujia78520@126.com)

'Department of General Surgery, Renji Hospital, School of Medicine, Shanghai Jiao Tong University, No. 160, Pujian Road, Shanghai 200127, P.R. China

${ }^{2}$ State Key Laboratory of Oncogenes and Related Genes, Shanghai Cancer Institute, Renji Hospital, School of Medicine, Shanghai Jiaotong University, Shanghai, P.R. China

These authors contributed equally: Site Yu, Yegian Zhang, Qing Li Edited by Z.-X. Xiao
}

that in patients with advanced gastric cancer, the incidence of liver metastasis is as high as $44 \%$, and the 5 -year survival rate of patients is approximately $10 \%^{6}$. Therefore, it is of utmost importance to study the mechanism of proliferation and metastasis of gastric cancer to improve the therapeutic effect and prognosis ${ }^{7,8}$.

CLDN6 is a member of the claudin family and the membrane protein encoded by the gene is a component of tight junction. The molecular weight of claudin protein is approximately $20-40 \mathrm{kDa}$. The common characteristics of claudin proteins include a short intracytoplasmic $\mathrm{N}$-terminal region, two extracellular ring domains formed by four transmembrane domains, and an intracytoplasmic C-terminal tail ${ }^{9}$. All claudin family members, except claudin12, have a carboxy-terminal PDZ binding motif that allows claudins to interact with cytoplasmic scaffolds ${ }^{10}$. The abnormal expression of CLDN6 is often associated with Hepatitis C infection ${ }^{11,12}$. However, there is sparse literature regarding its role in gastric cancer, and the mechanistic effect of claudin6 on tumorigenesis is still unclear. Epithelial mesenchymal transition (EMT) is an important process in tumorigenesis that is closely related

\section{(c) The Author(s) 2019}

(c) (i) Open Access This article is licensed under a Creative Commons Attribution 4.0 International License, which permits use, sharing, adaptation, distribution and reproduction cc) in any medium or format, as long as you give appropriate credit to the original author(s) and the source, provide a link to the Creative Commons license, and indicate if changes were made. The images or other third party material in this article are included in the article's Creative Commons license, unless indicated otherwise in a credit line to the material. If material is not included in the article's Creative Commons license and your intended use is not permitted by statutory regulation or exceeds the permitted use, you will need to obtain permission directly from the copyright holder. To view a copy of this license, visit http://creativecommons.org/licenses/by/4.0/. 
to tumor invasion, metastasis, and prognosis. Recent studies have shown that EMT plays an important role in many biological processes such as malignant proliferation of tumor cells, regulation of tumor microenvironment, and activation of tumor stem cells ${ }^{13-15}$. In addition, a previous study reported the correlation between claudin proteins and EMT ${ }^{16}$. However, the role of CLDN6 in the malignant transformation of gastric cancer through the EMT network is still unknown.

In the present study, we found that CLDN6 was significantly upregulated in gastric cancer, and its abnormal increased expression often predicted poor prognosis of GC patients. CLDN6 interacted with LATS1/2, resulting in the reduction of YAP1 phosphorylation, thereby increasing YAP1 nuclear translocation and activating downstream target genes. Moreover, YAP1 interacted with snail1 and promoted the process of EMT. CLDN6 therefore, might be a novel prognostic molecule for gastric cancer and a potential target candidate for future treatment of gastric cancer.

\section{Materials and methods}

\section{Patients and tissue samples}

In this study, gastric cancer tissues were obtained from 494 patients who underwent surgical resection in Renji hospital from 2010 to 2016. After surgical resection, all samples were immediately frozen in liquid nitrogen and stored at $-80^{\circ} \mathrm{C}$. The tissue samples were used to produce tissue microarrays for later use. The clinicopathological characteristics of the selected patients were obtained from their medical records. The guidelines published in the 7th edition of the TNM classification of tumors by the International Union Against Cancer (UICC) was used for histological classification of tumors according to the criteria set by the World Health Organization. This study was approved by the Ethics Committee of Renji Hospital and written informed consent was obtained from each patient.

\section{Cell lines and culture conditions}

The human gastric cancer cell lines MKN28, MKN45, HGC27, MGC803, BGC823, AGS, and human immortalized gastric epithelial cells GES1 were purchased from the Institute of Biochemistry and Cell Biology, Chinese Academy of Sciences, Shanghai, China. All the cells were cultured in RPMI 1640 medium (Invitrogen, Carlsbad, CA, USA) supplemented with $10 \%$ fetal bovine serum (FBS) and $1 \%$ penicillin/streptomycin $(\mathrm{P} / \mathrm{S})$.The cells were incubated in a humidified atmosphere at $37^{\circ} \mathrm{C}$ containing $5 \% \mathrm{CO}_{2}$.

\section{Lentiviral transfection}

Genomeditech (Shanghai, China) assisted in the design and production of CLDN6 shRNA. Gastric cancer cells were seeded in 6-well plates and incubated until cells were approximately $50 \%$ confluent. Depending on the MOI value of gastric cancer cells, appropriate lentivirus was added to each well. The cells were screened using antibiotics recommended by the company, and the transfection efficiency was measured by fluorescence quantitative PCR or western blotting. The target sequences of CLDN6 were sh-1, sense: $5^{\prime}$ GGGATTGTCTTTGTCATCTCA 3 '; sh-2, sense: 5' GAGTACCCTACCAAGAATTAC 3'; shcontrol, sense: 5' TTCTCCGAACGTGTCACGT 3'.

\section{RNA extraction and quantitative real-time PCR}

Total RNA was extracted using Trizol and reverse transcribed into cDNA by PrimeScriptTM (Takara Biomedical Technology, Beijing). Using $18 \mathrm{~S}$ rRNA as an internal control, real-time PCR analysis was performed on the 7500 real-time PCR system (Applied Biosystems), and the relative expression levels of target genes were calculated by the $-\Delta \Delta \mathrm{Ct}$ method. The primer sequences used are listed in Supplementary Table S1.

\section{Western blotting}

MKN28 and AGS gastric cancer cells were lysed using RIPA lysis buffer (Beyotime, Beijing, China) and protease inhibitors (Roche, CA, USA). The proteins were separated by $10 \%$ SDS polyacrylamide gel electrophoresis and subsequently transferred onto nitrocellulose (NC) membranes. The membranes were blocked in TBS buffer containing 5\% skim milk for $1 \mathrm{~h}$ at room temperature. After incubation with primary and secondary antibodies, ECL reagent was used to visualize the protein bands. CLDN6 (ab107059, Abcam), p-LATS1/2 (ab111344, Abcam), LATS1/2 (202761-AP, Proteintech), p-YAP1 (ab76252, Abcam), YAP1 (13584-1-AP 6900-1-Ig, Proteintech), snail1 (13099-1-AP, Proteintech), twist1(25465-1AP, Proteintech), zeb1 (21544-1-AP, Proteintech), Ecadherin (20874-1-AP, Proteintech), N-cadherin (GB111009, Servicebio), Vimentin (GB11192, Servicebio), Ki67 (GB13030, Servicebio), $\beta$-actin (GB11001, Servicebio), HRP (GB23301, GB23303, Servicebio), Lamin B1 (AB0054, Abways).

\section{Coimmunoprecipitation (Co-IP)}

Total protein extraction buffer (Beyotime, China) was used to extract proteins from MKN28 and AGS gastric cancer cells. Protein A/G magnetic beads (B23201, Bimake, China, Shanghai) were pre-incubated with antiYAP1 or anti-snail1 antibody on a spinning wheel at $4{ }^{\circ} \mathrm{C}$ for 30-60 min and washed 3 times. The antibody complex was then added to the protein solution. After the protein solution was fully combined with the magnetic bead-antibody complex, the complex was washed thrice with extraction buffer. The immunoprecipitate was 
collected by magnetic bead separation, heated, and western blotting was performed.

\section{ChIP assay}

ChIP assay was performed using the Pierce Agarose ChIP Kit (Thermo, 26156). Antibodies against snail1 (1:50, \#3879, Cell Signaling Technology) was used for IP. The primers used for the ChIP assay are listed in Supplementary Table S2. RNA was extracted from the immunoprecipitate and reverse transcribed into cDNA for PCR analysis. RNA enrichment was measured by RTPCR using primers specific for CDH1.

\section{Cell proliferation assay}

Proliferation capacity of gastric cancer cells was determined by the Cell Counting Kit 8 assay (CCK-8, Beyotime, China). After transfection with CLDN6 shRNA, gastric cancer cells were inoculated into 96-well plates (approximately 2000 cells per well). Cells were incubated with $10 \mu \mathrm{l}$ of CCK-8 reagent for $1 \mathrm{~h}$ in the dark, and optical density was measured at a wavelength of $450 \mathrm{~nm}$ using SpectrumMax Plus384 microplate reader (Molecular Devices). MKN28 and AGS gastric cancer cells (approximately 500 cells/well) were seeded in 6-well plates, and cultured for approximately 2 weeks. After washing twice in PBS, cells were fixed with $4 \%$ paraformaldehyde for $15 \mathrm{~min}$, and staining was performed by adding $0.5 \%$ crystal violet for $15 \mathrm{~min}$. The number of gastric cancer cell clones in different groups was calculated.

\section{Wound healing assay}

Approximately $5 \times 10^{5}$ cells were added to each well of a 6-well plate and cultured until completely confluent on the second day. Straight lines were drawn vertically on the monolayer with a sterile pipette tip. The cells were then washed with PBS for 3 times, and serum-free medium was added. Photographs were taken at $0,6,12$, and $24 \mathrm{~h}$.

\section{Transwell assay}

Transwell chamber was prepared, $600 \mu \mathrm{l}$ serum-free RPMI 1640 medium was added into the upper chamber, and $5 \times 10^{5}$ cells were seeded in each well. The culture medium containing $20 \%$ serum was added in the lower chamber. After $24 \mathrm{~h}$, the chamber was washed twice, $4 \%$ paraformaldehyde was used to fix the cells for $15 \mathrm{~min}$, and crystal violet was added for $15 \mathrm{~min}$ to stain the cells. The number of cells passing through the chamber was counted under the microscope.

\section{Immunohistochemistry and staining evaluation}

Immunohistochemistry (IHC) was performed as previously reported ${ }^{17}$. Paraffin sections were dewaxed using xylene, rehydrated by fractional ethanol, and antigens were extracted. The sections were blocked with $10 \%$ bovine serum albumin (BSA), incubated with primary antibody for $1 \mathrm{~h}$, and then incubated with secondary antibody for $30 \mathrm{~min}$ at room temperature. DAPI $(4,6-$ diamino-2-phenylindole hydrochloride; AppliChem, A4099) was used to stain the nucleus. Automated fluorescence microscope (Nikon) was used for imaging and analysis. The tissue sections were assessed and graded by two independent investigators who were unaware of the clinicopathological factors. The staining intensity scoring was 0 (negative), 1 (weak), 2 (medium), and 3 (strong).The degree of staining was stratified as $0(0 \%), 1(1-25 \%), 2$ (26-50\%), 3 (51-75\%), 4 (76-100\%), and defined as the percentage of positive staining area in the total tumor invasion area. The final score of CLDN6 expression was approximately $0-7$. The samples were divided into two groups: low CLDN6 expression (0-3 points) and high CLDN6 expression (4-7 points).

\section{Cellular immunofluorescence}

After immersion of the slides in $100 \%$ alcohol, residual alcohol was removed by flaming and ultraviolet irradiation. The glass slides were placed in a 6-well plate, and gastric cancer cells were added onto the glass slide, allowing the cells to grow up to $30-50 \%$ of the slide area on the second day. Cells were fixed with $4 \%$ paraformaldehyde for $15 \mathrm{~min}$ and treated with $0.5 \%$ Triton X100 for $1 \mathrm{~min}$. Non-specific binding sites were blocked by $1 \%$ BSA. The cells were incubated with the primary antibody for $1 \mathrm{~h}$ at room temperature, with the secondary antibody for $30 \mathrm{~min}$, and treated with DAPI for $30 \mathrm{~min}$ for nuclear staining. The cells were observed and analyzed using an automated fluorescence microscope (Nikon).

\section{Animal models}

To generate the subcutaneous tumor model in nude mice, $5 \times 10^{6}$ MKN18 cells were injected into the left axilla of each nude mouse. After 4 weeks, the nude mice were sacrificed, the subcutaneous tumor weight and volume were measured, and $4 \%$ paraformaldehyde was used to fix the tumor tissue. To generate the liver metastasis model, nude mice were anesthetized with $0.5 \%$ pentobarbital, the abdominal cavity was opened, and $1 \times$ $10^{6}$ BGC823 cells were injected into the spleen. After 4 weeks, the nude mice were sacrificed and liver metastases were observed. All tissues were fixed with $4 \%$ paraformaldehyde. All animal experiments were approved by the Ethics Committee of Renji Hospital.

\section{Statistical analysis}

The SPSS 22.0 software (SPSS Inc., Chicago, IL, USA) was used to analyze the data and mean \pm SD was calculated. Student's $t$ test and chi-square test were used in the study. Cox proportional hazard model was used for 
univariate and multivariate analyses to understand the factors affecting survival. Results with $p<0.05$ were considered as statistically significant.

\section{Results}

\section{CLDN6 is upregulated in gastric cancer}

Thirty-two pairs of matched gastric tissues from the TCGA dataset were selected for bioinformatics analysis. Differentially expressed genes were identified with the criteria set as $p<0.05$ and $\operatorname{logFC}>2$ (Fig. 1a).These genes were divided into upregulated and downregulated groups (Fig. 1b). Pathway analysis using the DAVID tool revealed genes that were closely related to cell-cell adhesion and cell junction (Fig. 1c). CLDN1, 2, 6, 9, 16, and 19 were found to be of significance in gastric cancer in the tight junction family, and CLDN6 expression was the highest (Fig. 1d). Subsequently, through fluorescence quantitative PCR, we found that CLDN6 was highly expressed in MKN28 cell line with increased malignancy in GC (Fig. 1e). CLDN6 expression in gastric cancer tissues was also significantly higher than adjacent normal tissues (Fig. 1f, g), and showed a similar pattern in the GEO datasets GSE26942 and GSE54129 (Fig. 1h, i). Therefore, these results suggested that CLDN6 played a pro-tumorigenic role in gastric cancer.

\section{Increased CLDN6 expression is associated with poor prognosis in GC patients}

To investigate if CLDN6 could predict the survival of gastric cancer patients, we analyzed the data from GEO

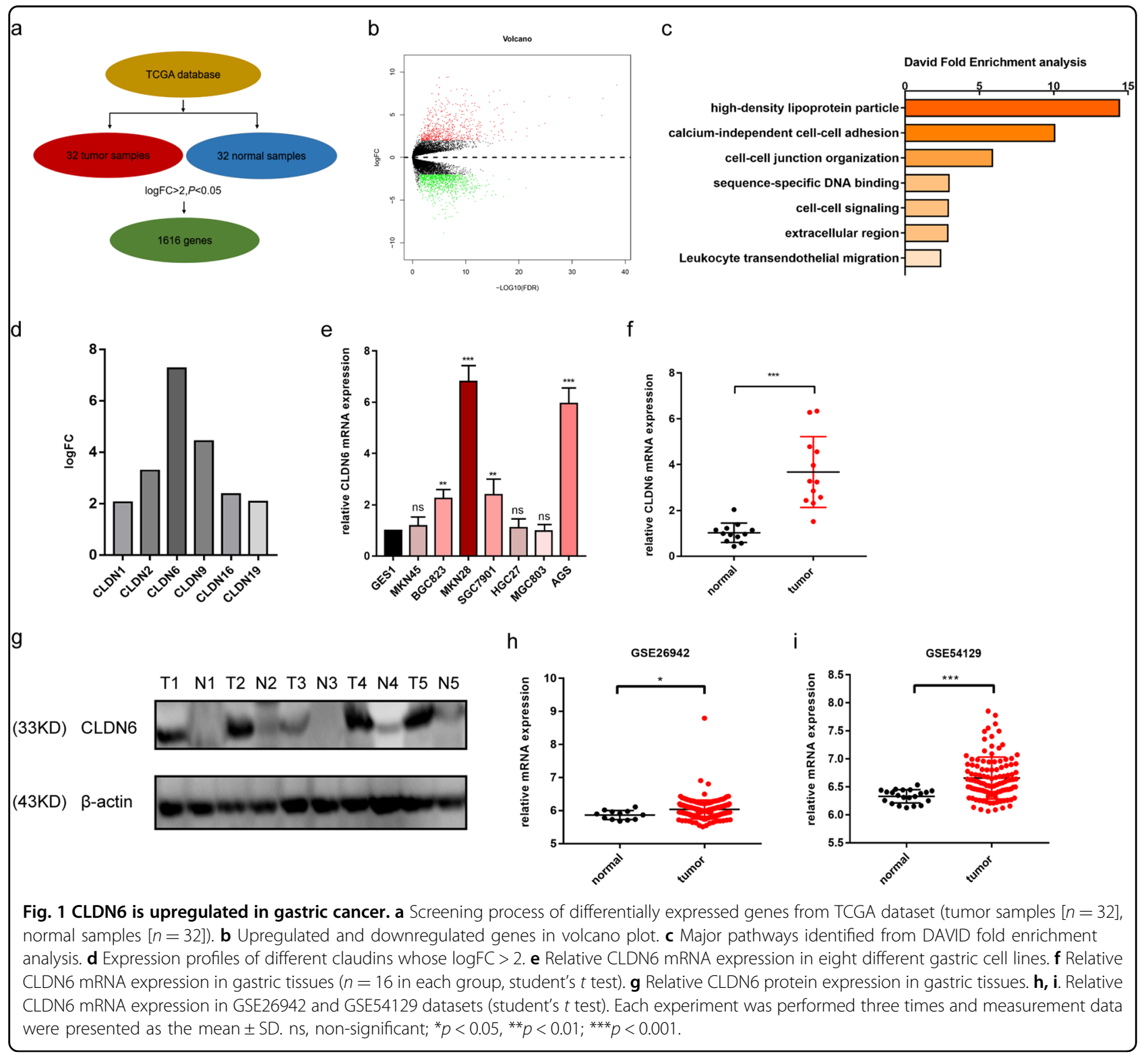




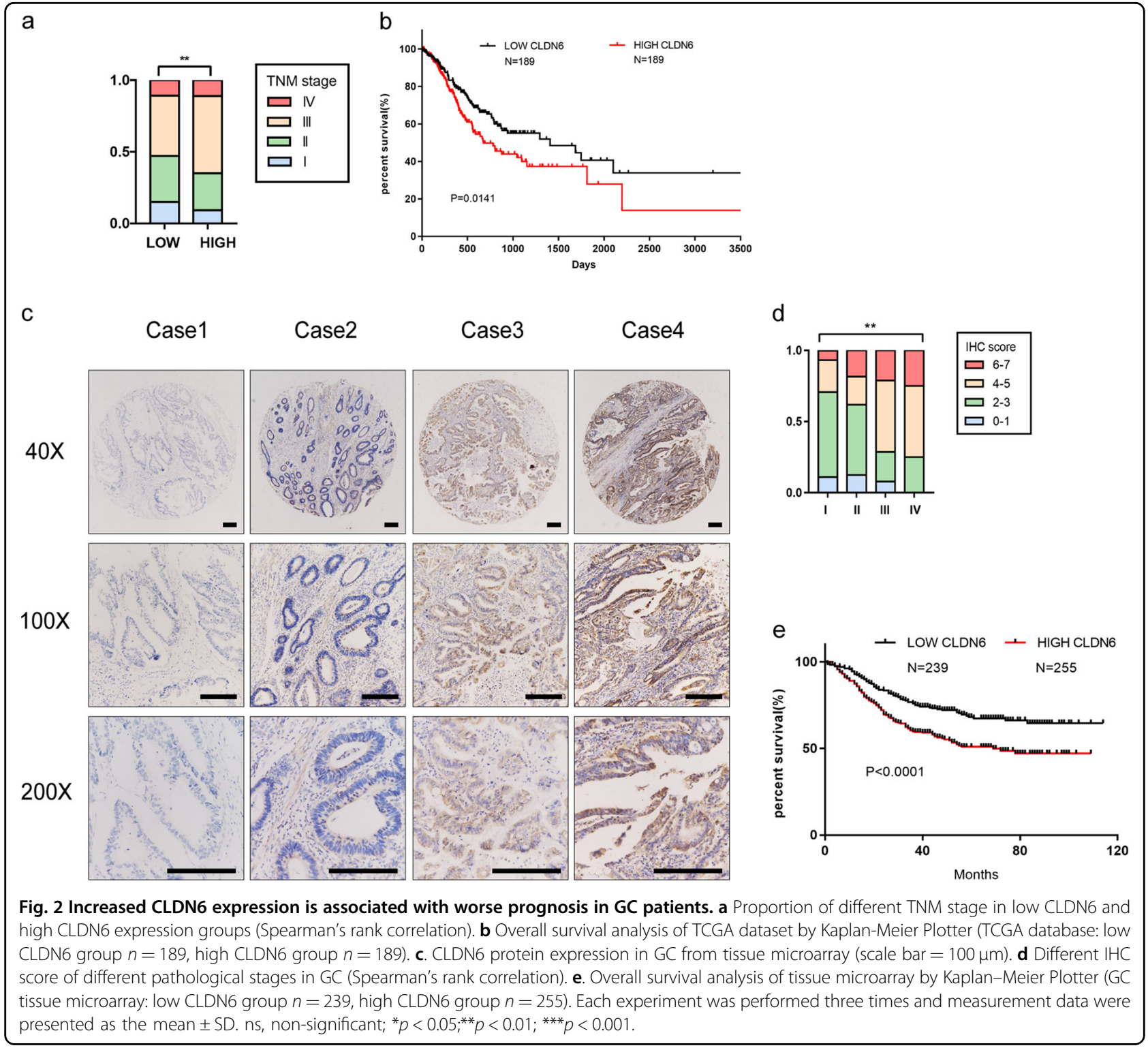

and TCGA databases as well as from Renji hospital. In TCGA database, when the patients were divided into two groups based on CLDN6 expression, we found that the number of patients with advanced-stage disease was more in high CLDN6 expression group (Fig. 2a). We also observed that the survival curve of GC patients with high CLDN6 expression indicated poor prognosis (Fig. 2b). Immunohistochemical staining for CLDN6 expression was performed on tissue microarray of 494 gastric cancer samples from Renji hospital (Fig. 2c). We found that the proportion of high staining score of CLDN6 increased gradually with TNM stage (Fig. 2d). The survival curve showed that patients with high expression of CLDN6 had worse prognosis (Fig. 2e). Moreover, we found that high CLDN6 expression was associated with age ( $>62$ years), tumor diameter, vessel carcinoma embolus, $\mathrm{T}$ stage, $\mathrm{N}$ stage and lymph node metastasis in GC patients (Table 1). Furthermore, univariate analysis revealed that tumor diameter, tumor embolus, nerve invasion, $\mathrm{T}$ stage, $\mathrm{N}$ stage, lymph node involvement, and high CLDN6 expression were risk factors in $\mathrm{GC}$, and multivariate analysis showed that tumor embolus and $\mathrm{T}$ stage were the major risk factors (Supplementary Table S3).

\section{CLDN6 promotes proliferation and invasion abilities of GC in vitro}

To further understand the biological function of CLDN6 in gastric cancer, shRNA was used to knockdown CLDN6 expression in MKN28 and AGS cells, and the knockdown efficiency was confirmed by fluorescence 
Table 1 The relationship between CLDN6 expression and clinical factors.

\begin{tabular}{|c|c|c|c|c|}
\hline \multirow[b]{2}{*}{ Parameters } & \multirow[b]{2}{*}{$n=494$} & \multicolumn{2}{|c|}{ CLDN6 expression } & \multirow[b]{2}{*}{$p$-value } \\
\hline & & Low $(n=239)$ & High $(n=255)$ & \\
\hline Age & & & & 0.012 \\
\hline$\leq 62$ & 247 & 134 & 113 & \\
\hline$>62$ & 247 & 105 & 142 & \\
\hline Gender & & & & 0.558 \\
\hline Male & 342 & 162 & 180 & \\
\hline Female & 152 & 77 & 75 & \\
\hline Tumor diameter & & & & $<0.001$ \\
\hline$\leq 4 \mathrm{~cm}$ & 255 & 143 & 112 & \\
\hline$>4 \mathrm{~cm}$ & 239 & 96 & 143 & \\
\hline Tumor embolus & & & & $<0.001$ \\
\hline No & 419 & 220 & 199 & \\
\hline Yes & 75 & 19 & 56 & \\
\hline Nerve invasion & & & & 0.215 \\
\hline No & 435 & 215 & 220 & \\
\hline Yes & 59 & 24 & 35 & \\
\hline N stage & & & & $<0.001$ \\
\hline No & 192 & 144 & 48 & \\
\hline N1-N3 & 302 & 95 & 207 & \\
\hline T stage & & & & $<0.001$ \\
\hline T0-T2 & 147 & 92 & 55 & \\
\hline T3-T4 & 347 & 147 & 200 & \\
\hline Node involvement & & & & $<0.001$ \\
\hline No & 197 & 145 & 52 & \\
\hline Yes & 297 & 94 & 203 & \\
\hline
\end{tabular}

Pearson $x^{2}$ test

quantitative PCR (Supplementary Fig. S1). The colony formation and CCK8 assays revealed that the proliferative ability of gastric cancer cells was inhibited after suppression of CLDN6 expression (Fig. 3a-c). Wound healing and transwell assays showed that the migration ability of gastric cancer cells significantly decreased upon CLDN6 knockdown (Fig. 3d-g). Together, these results suggest that silencing CLDN6 expression inhibited the proliferation and invasion abilities of GC in vitro.

\section{CLDN6 promotes proliferation and invasion abilities in GC} in vivo

To evaluate whether abnormal expression of CLDN6 enhanced tumorigenicity of gastric cancer in vivo, gastric cancer cells transfected with sh-CLDN6 and empty vector constructs, were injected subcutaneously and into the spleen of nude mice, respectively, to generate xenograft subcutaneous tumor model and liver metastasis model (Fig. 4a, e). The weight and volume of the transplanted tumors from nude mice were measured and the number of liver metastases from the liver metastasis model was calculated. The subcutaneous tumor weight and volume were significantly reduced in the sh-CLDN6 group (Fig. 4 a, b, d). Moreover, the sh-control group was prone to liver metastasis of gastric cancer, because the number of liver metastases in this group increased significantly (Fig. 4 e, f). Immunohistochemical staining of subcutaneous tumors showed that Ki67 expression in sh-CLDN6 group was significantly lower than sh-control group (Fig. 4c). In addition, strong E-cadherin and weak $\mathrm{N}$-cadherin staining was observed in sh-CLDN6 group (Fig. 4g). Collectively, these results indicated that high CLDN6 expression enhanced the proliferative and invasive abilities of tumors in vivo, which was consistent with our previous in vitro results.

\section{CLDN6 promotes YAP1 nuclear translocation that interacts with snail1 to promote EMT}

Our in vitro and in vivo experimental results revealed that the abnormal expression of CLDN6 was related to the proliferation and invasion abilities of gastric cancer. Therefore, we explored the possible mechanism of CLDN6 in promoting the progression of gastric cancer. The GSE110875 dataset contained 80 gastric cancer samples, which were divided into low and high CLDN6 expression groups. We then used GSEA to analyze the changes in related pathways, and identified the Hippo signaling pathway to be of significance (Fig. 5a). The immunoprecipitation assay results showed that CLDN6 and LATS1/2 interacted with each other (Fig. 5b), thus causing phosphorylation and dephosphorylation changes in downstream YAP1. This interaction became weak after we silenced CLDN6 expression (Fig. 5c). In MKN28 and AGS cell lines, western blot assay showed that p-LATS and p-YAP1 levels were increased significantly in the shCLDN6 group (Fig. 5d).The increased levels of p-YAP1 led to decreased YAP1 entry into the nucleus, thereby inhibiting the development of gastric cancer (Fig. 5e). We also observed through cellular immunofluorescence assay and nuclear extraction experiment that, compared to the sh-CLDN6 group, sh-control group showed significantly increased expression of YAP1 in the nucleus(Fig. 5e-g) and activated the expression of downstream target genes such as CYR61, CTGF, AREG, and AMOTL2 (Fig. 5k). Because our previous experiments showed that reduced CLDN6 expression affected the invasion ability of gastric cancer cells, we speculated that CLDN6 was closely associated with the process of EMT. Western blotting results showed that knockdown of CLDN6 expression 


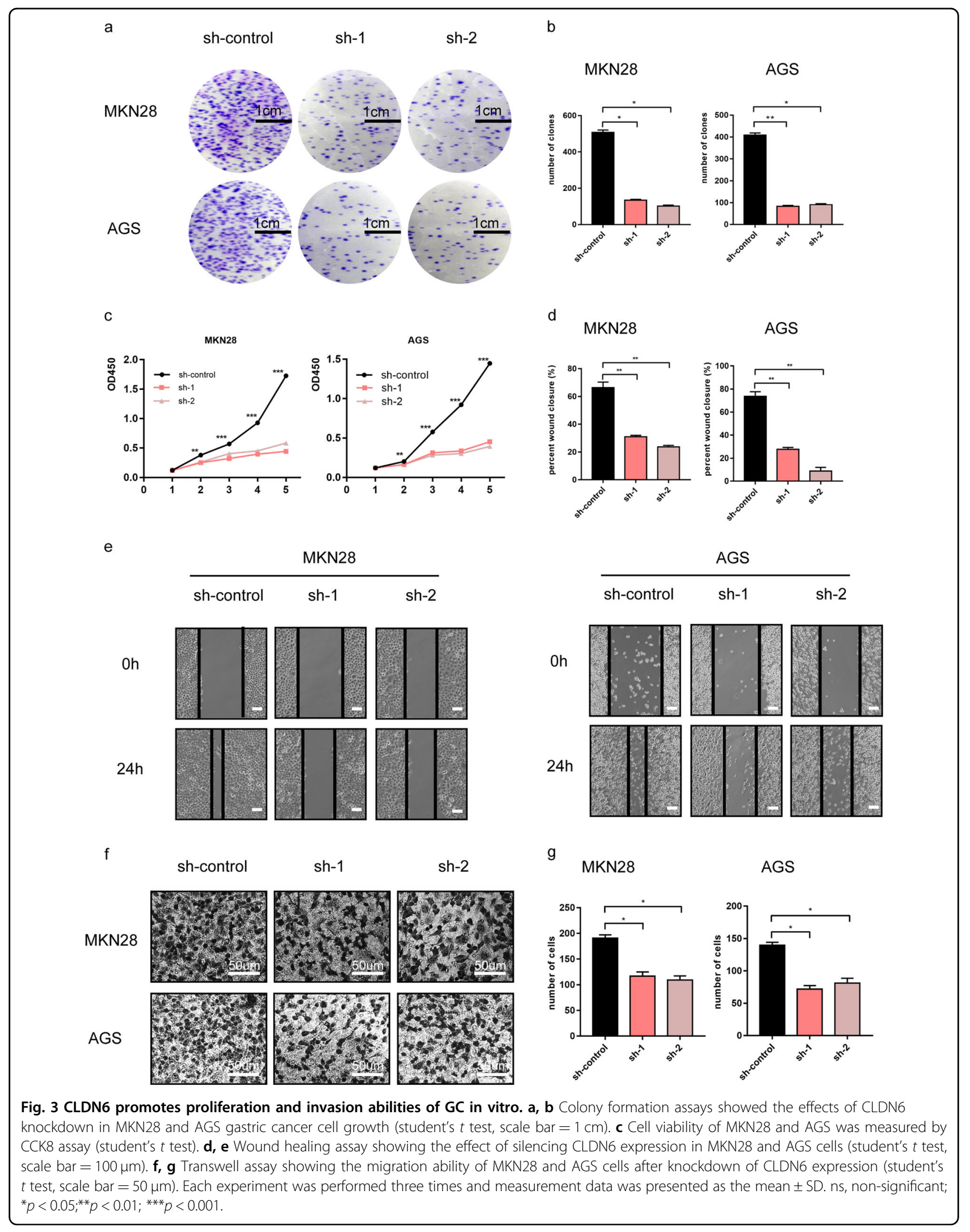



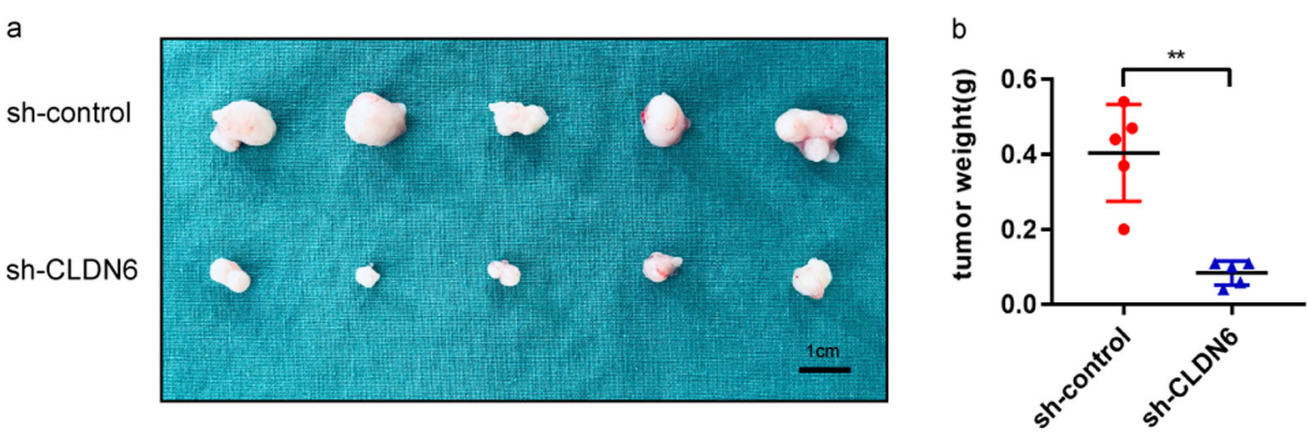

c
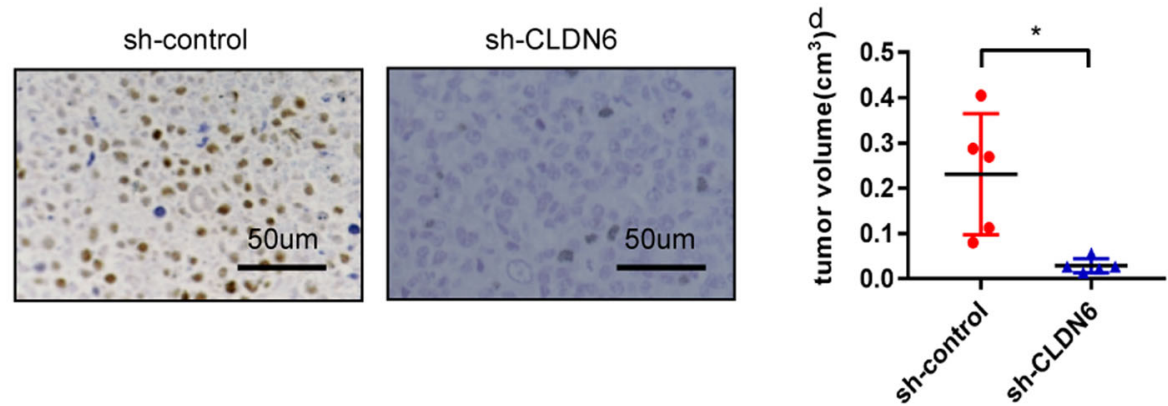

e ki67
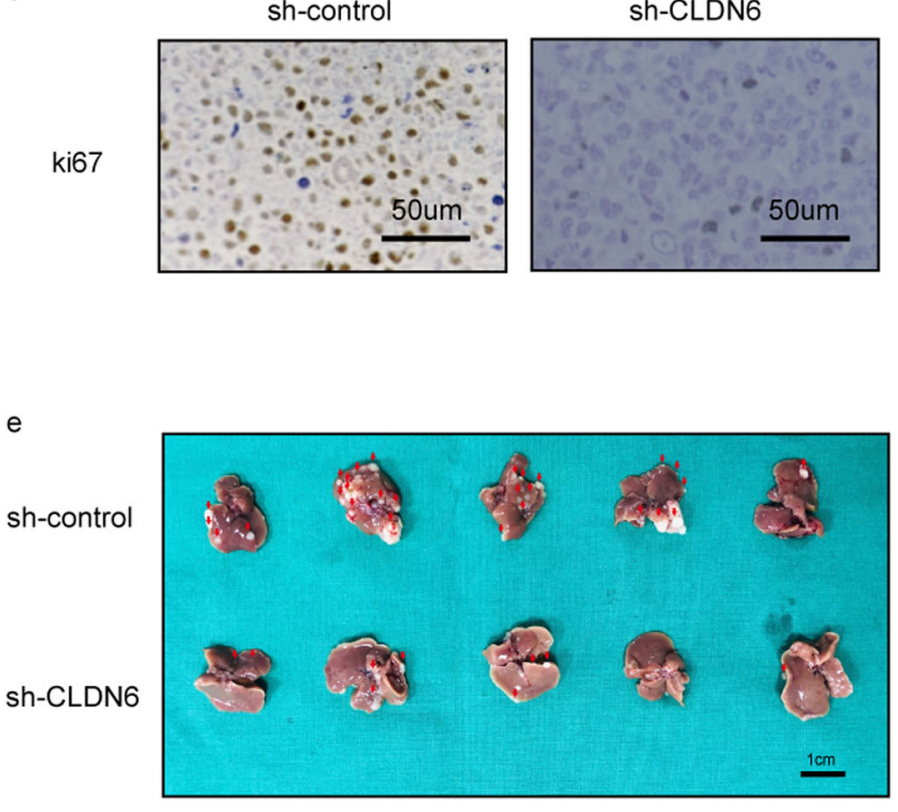

f

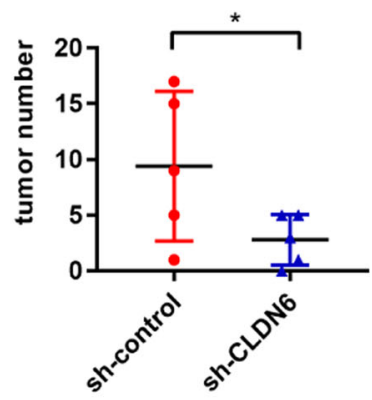

g

liver

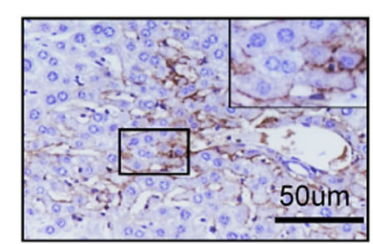

E-cadherin

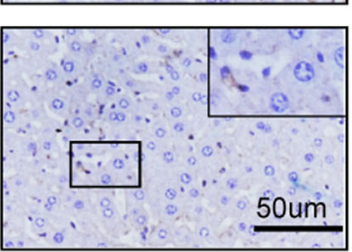

sh-control
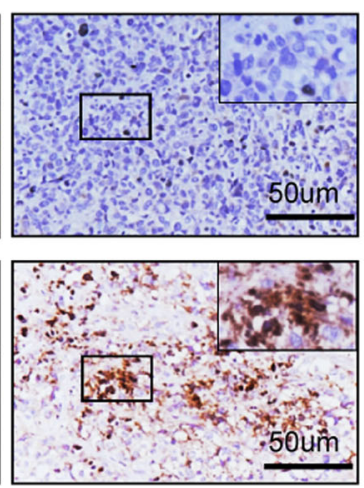

sh-CLDN6
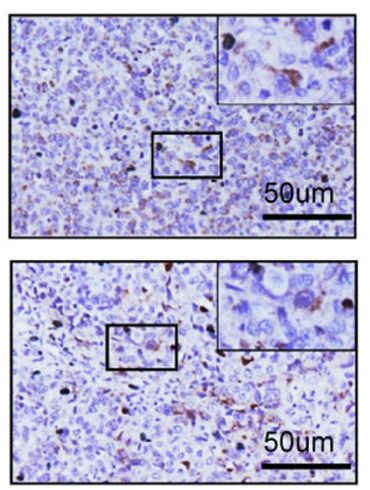

Fig. 4 CLDN6 promotes proliferation and invasion abilities of GC in vivo. a Nude mice were injected with sh-CLDN6 or sh-control vector transfected cells (sh-CLDN6 $[n=5]$ or sh-control $[n=5]$, scale bar $=1 \mathrm{~cm}$ ). b Tumor weight of sh-control and sh-CLDN6 groups (student's $t$ test). c Subcutaneous tumors were stained with Ki67 in sh-CLDN6 and sh-control group (scale bar $=50 \mu \mathrm{m}$ ). d Tumor volume of sh-control and sh-CLDN6 groups (student's $t$ test). e Tumor growth assessment in liver metastasis model of GC (red arrows indicate metastasis, scale bar $=1 \mathrm{~cm}$ ). $\mathbf{f}$ Tumor numbers in sh-control and sh-CLDN6 groups in liver metastasis model of GC ( $n=5$ in each group, student's $t$ test). $\mathbf{g}$ E-cadherin and $\mathrm{N}$-cadherin staining of adjacent normal tissues (liver tissue group) and tumor tissues (sh-CLDN6 and sh-control groups, scale bar $=50 \mu m$ ). Each experiment was performed three times and measurement data was presented as the mean $\pm \mathrm{SD}$. ns, non-significant; ${ }^{*} p<0.05 ;{ }^{* *} p<0.01 ;{ }^{* * *} p<0.001$. 


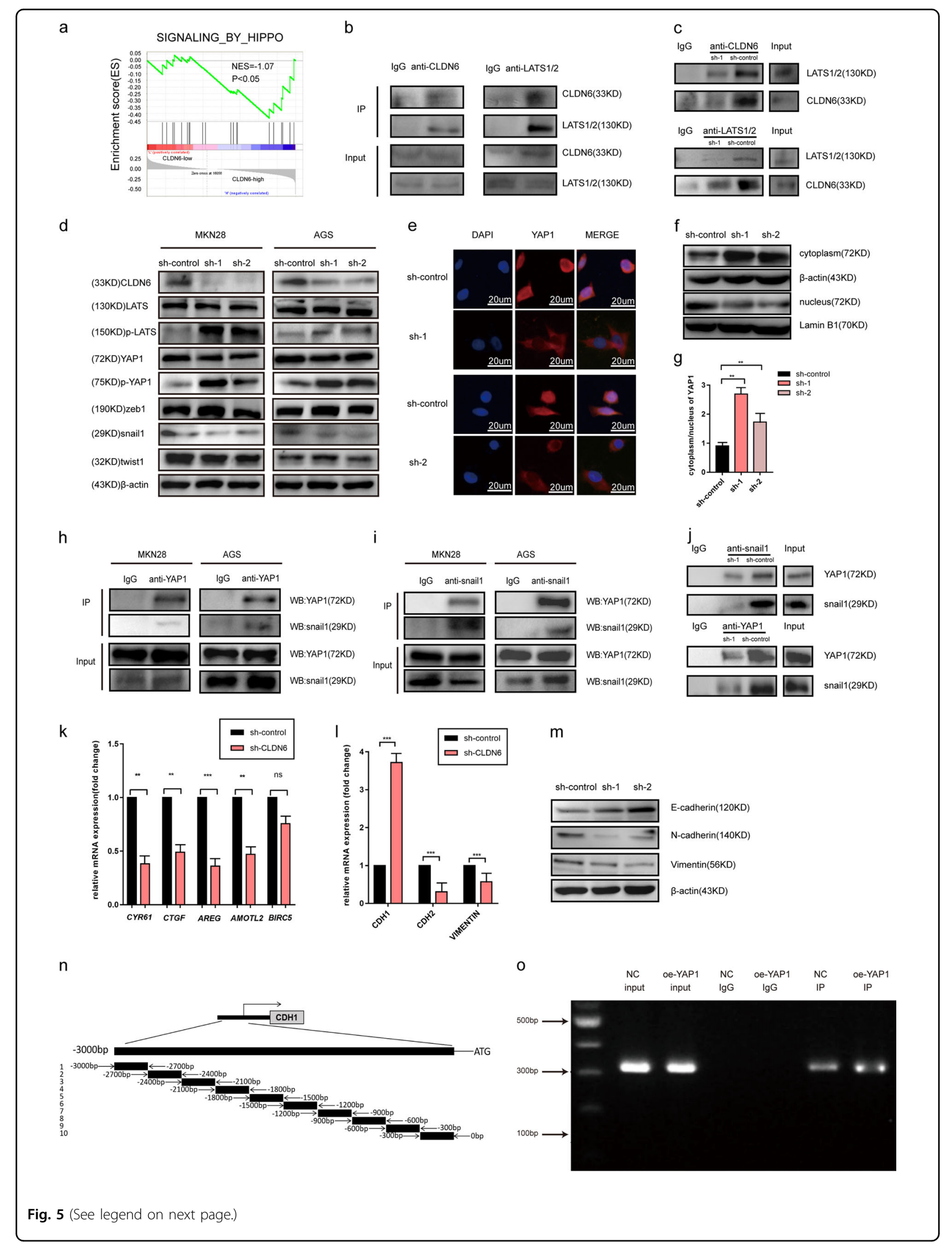


(see figure on previous page)

Fig. 5 CLDN6 promotes YAP1 nuclear translocation that interacts with snail1 to promote the process of EMT. a GSEA analysis was performed for CLDN6 expression in GC, including low CLDN6 expression group (40 samples) and high CLDN6 expression group (40 samples) from GSE110875. b, $\mathbf{c}$ Immunoprecipitation assay showed the interaction between CLDN6 and LATS1/2. d Protein levels of CLDN6, LATS, p-LATS, YAP1, p-YAP1, zeb1, snail1, twist1, and $\beta$-actin were measured in MKN28 and AGS cells by western blotting. e Cellular immunofluorescence assay showed YAP1 localization in MKN28 GC cells in sh-control group and sh-CLDN6 group (scale bar $=20 \mu \mathrm{m}$ ). $\mathbf{f}, \mathbf{g}$ Nuclear extraction experiment showed the quantification of the cytoplasm/nucleus of YAP1. $\mathbf{h}, \mathbf{i}, \mathbf{j}$ Immunoprecipitation assay showed the interaction between YAP1 and snail1 in MKN28 and AGS cells. $\mathbf{k}$ mRNA levels of downstream genes of YAP1 in sh-control and sh-CLDN6 groups by RT-PCR. I mRNA levels of CDH1, CDH2, and Vimentin in sh-control and sh-CLDN6 groups by RT-PCR. $\mathbf{m}$ Protein levels of E-cadherin, N-cadherin, and Vimentin in sh-control and sh-CLDN6 groups by western blotting. $\mathbf{n}$ Schematic of primer design for $\mathrm{CDH} 1$ promoter sequences. Ten primer sets with a 300-bp partition were designed for PCR to test the direct binding of YAP1-snail1 to the CDH1 promoter and the primer pairs produced 10 fragments of $300 \mathrm{bp}$. o Amplification of CDH1 promoter sequence from ChIP DNA validated the binding of YAP1-snail1 to the $\mathrm{CDH} 1$ promoter site. Every experiment was performed three times and measurement data was presented as the mean \pm SD. ns, non-significant; ${ }^{*} p<0.05 ;{ }^{* *} p<0.01 ;{ }^{* * *} p<0.001$

caused significant changes in snail1 protein, while there were no significant alterations in zeb1 and twist1 (Fig. 5d). Therefore, we reasonably speculated that CLDN6 might promote EMT through the YAP1-snail1 axis. Interestingly, the coimmunoprecipitation assay confirmed the interaction between YAP1 and snail1 (Fig. $5 \mathrm{~h}$, i) and, YAP1 did not interact with either twist1 or zeb1 (Supplementary Fig. S3) in MKN28 and AGS cells. The interaction between YAP1 and snail1 became weak after we silenced CLDN6 expression (Fig. 5j). GC cells were transfected with sh-CLDN6, and protein and mRNA levels of EMT markers were evaluated. Results showed that the expression level of E-cadherin significantly increased, while the levels of $\mathrm{N}$-cadherin and vimentin significantly decreased in sh-CLDN6 group (Fig. $5 \mathrm{l}, \mathrm{m}$ ). Subsequently, ChIP assay was performed and we observed that the YAP1-snail1 complex indeed bound to the promoter of CDH1 at approximately $-300 \mathrm{bp}$ and, when we overexpressed YAP1, the expression was stronger than before (Fig. $5 \mathrm{n}$, o). These results indicated that CLDN6 activated the expression of related oncogenes by increasing YAP1 nuclear translocation, thus accelerating the proliferation of GC cells. In addition, the interaction between YAP1 and snail1 promoted EMT of gastric cancer cells, thereby promoting invasion of gastric cancer.

\section{Overexpression of YAP1 partially rescued the diminished proliferation and invasion abilities caused by CLDN6 knockdown}

To further understand the CLDN6-YAP1-snail1 axis, we overexpressed YAP1S127A (one of the phosphorylation sites of YAP1 was mutated) in MKN28 and AGS cells that were transfected with sh-CLDN6 previously. Western blot analysis showed that after the overexpression of YAP1 in sh-CLDN6 group, the expression of LATS1/2 upstream remained unchanged, while the expression of snail1 was relatively increased downstream (Fig. 6a). Overexpression of YAP1 partially rescued the effect of diminished expression of CLDN6 on snail1. Subcutaneous tumor model showed that suppression of CLDN6 expression reduced tumor load, while overexpression of YAP1 reversed this trend of tumor load reduction (Fig. 6 c, e and $\mathrm{f})$. In addition, in the liver metastasis model, liver tissues were more likely to have metastasis in the shCLDN6 + oe-YAP1 group compared to the sh-CLDN6 group (Fig. 6d). Moreover, immunohistochemical staining was performed to evaluate the expression of Ki67, Ecadherin, and N-cadherin. We found that overexpression of YAP1 partially reversed the tumor-inhibiting effect CLDN6 knockdown. (Fig. 6 b, g).To further evaluate the effect of CLDN6-YAP1-snail1 axis, we again overexpressed snail1 in sh-CLDN6 GC cells. We found that snail1 overexpression partially increased the cell invasion ability but not the proliferation ability (Fig. $6 \mathrm{~h}-\mathrm{j}$ ). Based on these results, we speculated that YAP1 was able to moderately rescue the diminished effects caused by CLDN6 knockdown and CLDN6-YAP1-snail1 axis affected the proliferation and invasion abilities of gastric cancer.

\section{Discussion}

Claudin proteins play critical roles in human tumors, affecting cell proliferation, differentiation, migration, metastasis, apoptosis, and other processes ${ }^{18-21}$. For instance, claudin 18 was found to regulate lung stem cell and progenitor cell heterogeneity, and tumor formation by affecting YAP activity ${ }^{22}$. A previous study also demonstrated that claudin 18 could regulate the proliferation of pulmonary epithelial cells ${ }^{23}$. Another study revealed that downregulation of claudin3 could highly activate the Wnt/ $\beta$-catenin and IL6/gp130/Stat3 signaling pathways, and enhance the malignancy of colorectal cancer $^{24}$. It has been suggested that claudin 2 on the surface of tumor cells could interact with liver cells to promote liver metastasis of breast cancer ${ }^{25}$. These studies largely reflected the role of claudin proteins in the process of tumorigenicity by activating or inactivating downstream signaling pathways, which were closely related to 


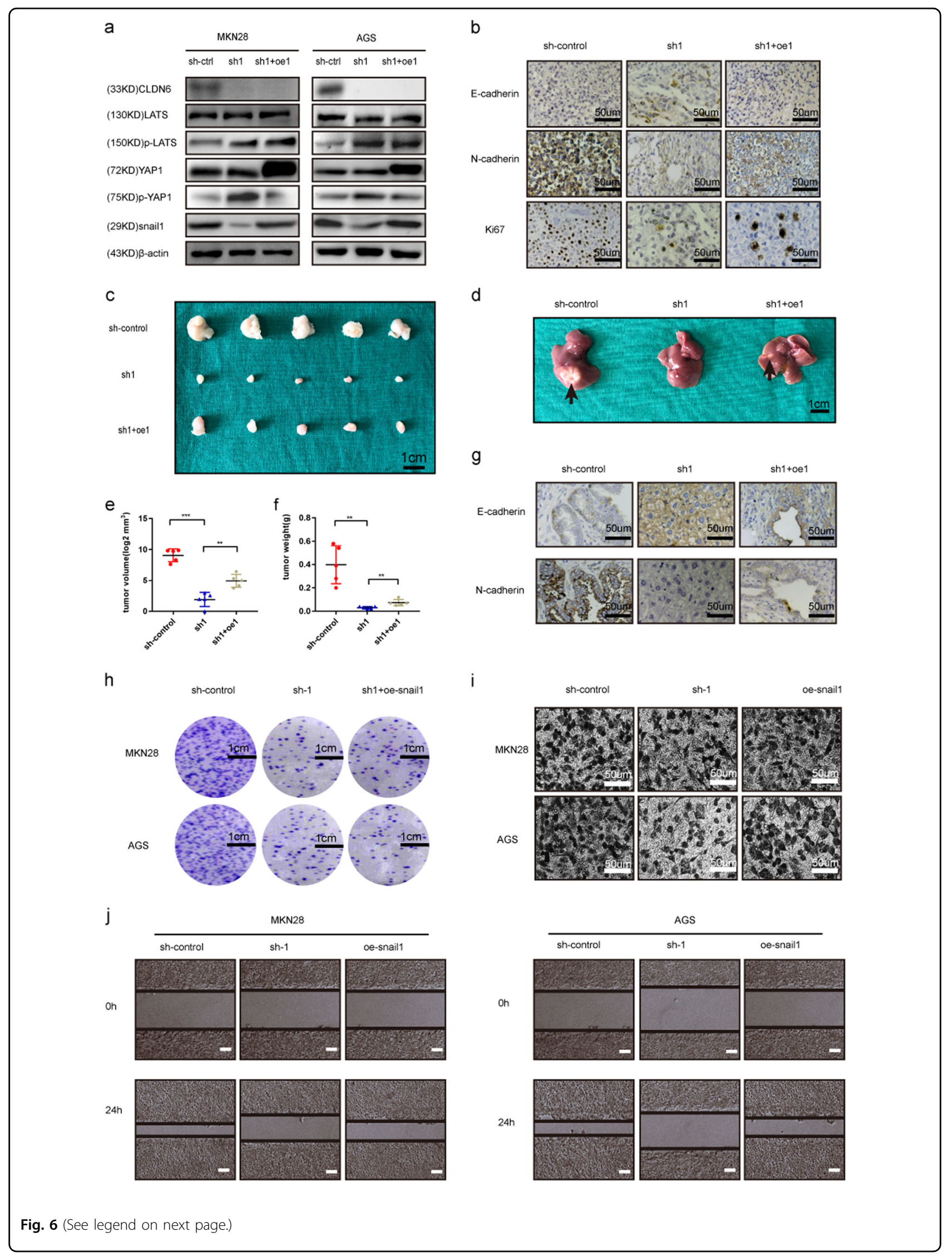


(see figure on previous page)

Fig. 6 Overexpression of YAP1 partially rescued the diminished proliferative and invasive ability caused by silencing CLDN6 expression. a Protein levels of CLDN6, LATS, p-LATS, YAP1, p-YAP1, snail1, and $\beta$-actin were measured in MKN28 and AGS cells by western blotting in sh-control, sh-CLDN6 and sh-CDLN6 + oe-YAP1S127A group. b Subcutaneous tumors in each group were stained with E-cadherin, N-cadherin and Ki67 (scale $\mathrm{bar}=50 \mu \mathrm{m}$ ). $\mathbf{c}$ Nude mice were injected with sh-control vector, sh-CLDN6 and sh-CDLN6 + oe-YAP1S127A transfected cells (sh-control [ $n=5]$, shCLDN6 group $[n=5]$, sh-CDLN6 + oe-YAP1S127A $[n=5]$, scale bar $=1 \mathrm{~cm}$ ). d Tumor growth assessment in liver metastasis model of GC in different groups ( $n=3$ in each group, black arrows show metastasis, scale bar $=1 \mathrm{~cm}$ ). e, $\mathbf{f}$ Tumor volume and weight of sh-control, sh-CLDN6, and shCDLN6 + oe-YAP1S127A groups (student's $t$ test). $\mathbf{g}$ Liver metastatic tissues were stained for E-cadherin or N-cadherin (sh-CLDN6 group had no liver metastasis, scale bar $=50 \mu \mathrm{m}$ ). $\mathbf{h}$ Colony formation assay showed the effects of snail1 overexpression on MKN28 and AGS gastric cancer cell growth (student's $t$ test, scale bar $=1 \mathrm{~cm}$ ). i Transwell assay showed the migration ability of MKN28 and AGS cells after snail1 overexpression (student's $t$ test, scale bar $=50 \mu \mathrm{m}$ ). $\mathbf{j}$ Wound healing assay showed the influence of snail 1 overexpression on MKN28 and AGS cells (student's $t$ test, scale bar $=$ $100 \mu \mathrm{m})$. Every experiment was performed three times and measurement data was presented as the mean $\pm \mathrm{SD}$. ns, non-significant; ${ }^{*} p<0.05 ;{ }^{* *} p<$ $0.01 ;{ }^{* *} p<0.001$.

epithelial cell homeostasis, invasion, chronic inflammation, and cancer. Claudin proteins could also promote EMT through zeb-1/E-cadherin, Wnt signaling, MMP9/ Notch signaling, and other signaling pathways ${ }^{26}$. In this study, we found that claudin 6 is highly expressed in gastric cancer, especially in a cell and tissue type specific manner with high malignancy potential. The abnormal expression of claudin6 resulted in EMT, indicating poor prognosis. Further, through GSEA analysis, we found that the aberrant expression of claudin6 was closely associated with the Hippo signaling pathway. The upstream membrane protein of the Hippo signaling pathway senses the extracellular growth inhibition signal and activates a kinase cascade resulting in a series of phosphorylation reactions, and finally phosphorylates the downstream effectors YAP1 and TAZ ${ }^{27-29}$. Cytoskeletal proteins bind to phosphorylated YAP1 and TAZ, retaining them in the cytoplasm and thereby reducing their nuclear activity ${ }^{30-32}$. Essentially, YAP1 is associated with the process of EMT. There have been studies reporting that the interaction between KRAS and YAP1 could regulate EMT and tumor survival ${ }^{33}$. In addition, the self-renewal of liver cells was dependent on YAP1TGF- $\beta$-induced $\mathrm{EMT}^{34}$. It has been reported that the Helicobacter pylori CagA protein promoted the process of EMT in gastric cancer by triggering the tumorigenic YAP pathway ${ }^{35}$. In this study, we found that claudin6 reduced the phosphorylation level of downstream YAP1 by interacting with LATS1/2, thereby increasing the nuclear activity of YAP1. Subsequently, immunoprecipitation assay showed that YAP1 interacted with snail1 and influenced the expression of downstream target genes such as $C D H 1, C D H 2$, and Vimentin, thereby causing EMT in gastric cancer. Claudins play an important role in cancer development and the corresponding claudin monoclonal antibodies designed to treat cancer are expected to be highly specific, which help in reduce the side effects of chemotherapy and thereby improve treatment efficacy. Currently, monoclonal antibodies have been produced for CLDN1, CLDN2, CLDN3, CLDN4, CLDN6, and CLDN18.2 ${ }^{26}$. IMAB027, a monoclonal antibody against CLDN6, has been tested in phase I/II trials for ovarian cancer ${ }^{36}$. If the monoclonal antibody therapy shows promise, efforts are required to extrapolate this strategy to treat gastric cancer.

There are still certain limitations in this study. First, in the animal model of liver metastasis, we found that the nude mice in sh-CLDN6 group were less prone to liver metastasis than the sh-control group. So far, we have little understanding of the mechanism by which CLDN6 promotes liver metastasis in GC. As reported in a previous study, claudins on tumor cells interacted with claudins in liver cells, promoting cancer cell growth in liver tissues. The process of liver metastasis involves a complex mechanism, which requires further exploration. Second, although the immunoprecipitation assay results showed that CLDN6 interacted with LATS1/2, the way their interaction affected the downstream proteins is unclear. We have tested the protein levels of LATS, p-LATS, YAP1, and p-YAP1 by western blot analysis. We speculated that the interaction of LATS1/2 with CLDN6 relatively decreased the conversion of LATS into p-LATS, thereby inhibiting the Hippo signaling pathway. Upon CLDN6 knockdown, the interaction between CLDN6 and LATS was diminished, which led to an increase in p-LATS thus activating the Hippo signaling pathway (Fig. 7). However, an in-depth study needs to be carried out to elucidate these mechanisms.

In summary, the present study found the protumorigenic effect of CLDN6 in gastric cancer and revealed that CLDN6 promoted YAP1 nuclear translocation by reducing YAP1 phosphorylation, thereby activating downstream oncogenes. It promoted EMT of gastric cancer through YAP1-snail1 interaction, thus enhancing the proliferation and invasion abilities of 


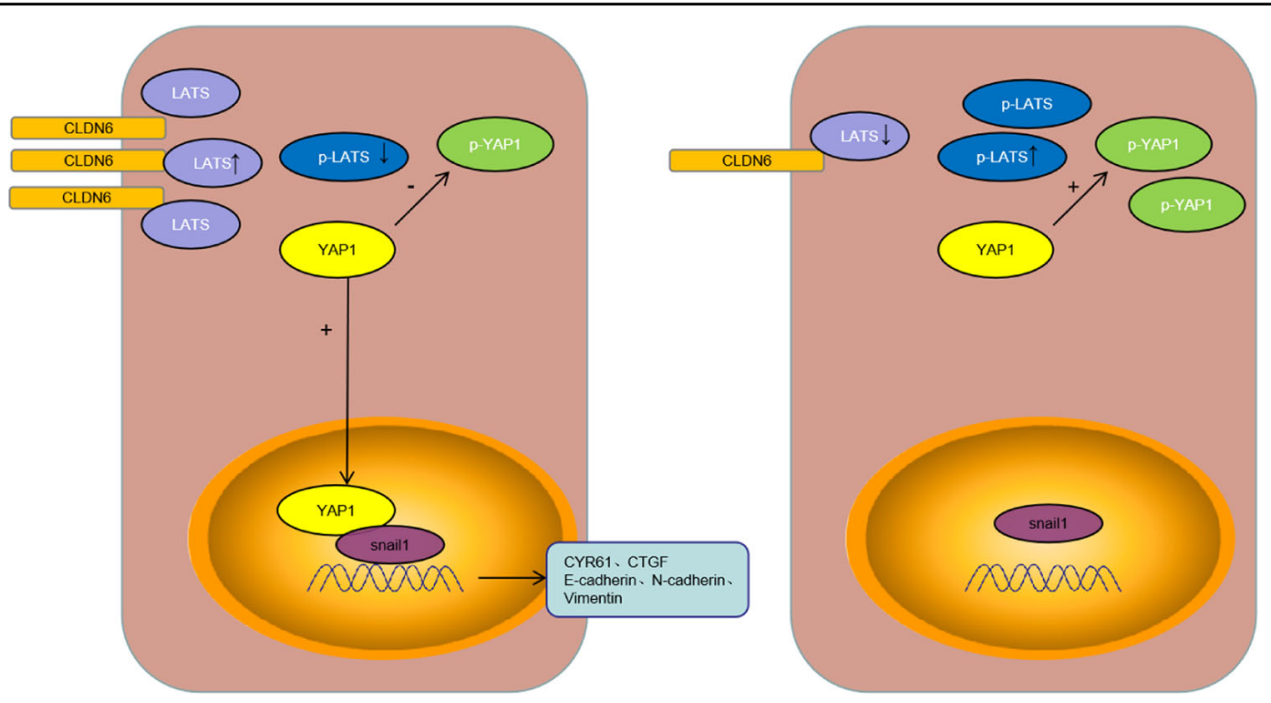

Fig. 7 Schematic representation of the mechanism of CLDN6-YAP1-snail1 axis. Upregulated CLDN6 expression: CLDN6 interacted with LATS, reducing the conversion of LATS into p-LATS, and YAP1 into p-YAP1. Therefore, increased amount of YAP1 entered cell nucleus to activate its downstream target genes. Increased YAP1 interacted with snail1 to bind to the promoter of EMT related genes to enhance EMT progression. Downregulated CLDN6 expression: Decreased amount of CLDN6 interacted with LATS thereby increasing p-LATS and resulting in increased p-YAP1. Due to decreased levels of YAP1 in the cell nucleus, the downstream pathways were inhibited.

gastric cancer cells. This study provides new insights to design relevant therapeutic strategies for the treatment of gastric cancer.

\section{Acknowledgements}

This study was supported by the National Natural Science Foundation of China (No.31872740).

\section{Conflict of interest}

The authors declare that they have no conflict of interest.

\section{Publisher's note}

Springer Nature remains neutral with regard to jurisdictional claims in published maps and institutional affiliations.

Supplementary information accompanies this paper at (https://doi.org/ 10.1038/s41419-019-2168-y).

Received: 20 July 2019 Revised: 19 November 2019 Accepted: 20 November 2019

Published online: 11 December 2019

\section{References}

1. Ferlay, J. et al. Cancer incidence and mortality worldwide sources, methods and major patterns in GLOBOCAN 2012. Int J. Cancer 136, E359-E386 (2015).

2. Torre, L. A. et al. Global cancer statistics, 2012. CA Cancer J. Clin. 65, 87-108 (2015).

3. Siegel, R., Naishadham, D. \& Jemal, A. Cancer statistics. Cancer J. Clin. 62, 10-29 (2012).

4. Chen, W. et al. Cancer statistics in China, 2015. CA Cancer J. Clin. 66, 115-132 (2016).

5. Obenauf, A. C. \& Massagué, J. Surviving at a distance:organ-specific metastasis. Trends Cancer 1, 76-91 (2015).

6. Shitara, K. et al. Reporting patient characteristics and stratification factors in randomized trials of systemic chemotherapy for advanced gastric cancer. Gastric Cancer 15, 137-143 (2012).
7. Digklia, A. \& Wagner, A. D. Advanced gastric cancer: current treatment landscape and future perspectives. World J. Gastroenterol. 22, 2403-2414 (2016).

8. Ferro, A. et al. Worldwide trends in gastric cancer mortality (1980-2011), with predictions to 2015, and incidence by subtype. Eur. J. Cancer 50, 1330-1344 (2014).

9. Lal-Nag, M. \& Morin, P. J. The claudins. Genome Biol. 10, 235 (2009).

10. Ruffer, C. \& Gerke, V. The C-terminal cytoplasmic tail of claudins 1 and 5 but not its PDZ-binding motif is required for apical localization at epithelial and endothelial tight junctions. Eur. J. Cell Biol. 83, 135-144 (2004).

11. Zheng, A. et al. Claudin-6 and Claudin-9 function as additional coreceptors for hepatitis C virus. J. Virol. 81, 12465-12471 (2007).

12. Haid, $\mathrm{S}$. et al. Isolate-dependent use of claudins for cell entry by hepatitis $C$ virus[J]. Hepatology 59, 24-34 (2014).

13. Micalizzi. et al. The Six 1 homeoprotein induces human mammary carcinoma cells to undergo epithelial-mesenchymal transition and metastasis in mice through increasing TGF-beta signaling. J. Clin. Invest. 119, 2678-2690 (2009).

14. Rhim et al. EMT and dissemination precede pancreatic tumor formation. Cell 148, 349-361 (2012).

15. Heinrich. et al. The inflammatory tumor microenvironment, epithelial mesenchymal transition and lung carcinogenesis. Cancer M icroenviron. 5, 5-18 (2012).

16. Ching, F. L. et al. Snail-induced claudin-11 prompts collective migration for tumour progression. Nat. Cell Biol. 21, 251-262 (2019).

17. $\mathrm{Hu}, \mathrm{L}$. P. et al. Targeting Purinergic Receptor P2Y2 prevents the growth of pancreatic ductal adenocarcinoma by inhibiting cancer cell glycolysis[]]. Clin. Cancer Res. 10, 1158/1078-0432 (2018).

18. Singh, A. B. \& Dhawan, P. Claudins and cancer: Fall of the soldiers entrusted to protect the gate and keep the barrier intact. Semin. Cell Dev. Biol. 42, S1084952115000981 (2015).

19. Kwon, M. J. et al. Derepression of CLDN3 and CLDN4 during ovarian tumorigenesis is associated with loss of repressive histone modifications. Carcinogenesis 31, 974 (2010).

20. Chang, T. L. et al. Claudin-1 has tumor suppressive activity and is a direct target of RUNX3 in gastric epithelial cells. Gastroenterology 138, 255-265.e3 (2010).

21. Mi, J. K. Emerging roles of claudins in human cancer. Int. J. Mol. Sci. 14 18148-18180 (2013).

22. Zhou, B. et al. Claudin-18-mediated YAP activity regulates lung stem and progenitor cell homeostasis and tumorigenesis. J. Clin. Investig. 128, 3 (2018).

23. Kotton, D. N. Claudin-18: unexpected regulator of lung alveolar epithelial cell proliferation. J. Clin. Investig. 128, 903-905 (2018). 
24. Ahmad, R. et al. Loss of claudin-3 expression induces IL6/gp130/ Stat3 signaling to promote colon cancer malignancy by hyperactivating Wnt/ $\beta$-catenin signaling. Oncogene 36, 47 (2017).

25. Tabariès, $\mathrm{S}$. et al. Claudin-2 promotes breast cancer liver metastasis by facilitating tumor cell interactions with hepatocytes. Mol. Cell Biol. 32, 2979-2991 (2012).

26. Zeisel, M. B. et al. Tight junction proteins in gastrointestinal and liver disease. Gut https://doi.org/10.1136/gutjnl-2018-316906 (2018).

27. Yu, F. X., Zhao, B. \& Guan, K. L. Hippo pathway in organ size control, tissue homeostasis, and cancer. Cell 163, 811-828 (2015).

28. Zanconato, F., Cordenonsi, M. \& Piccolo, S. YAP/TAZ at the roots of cancer Cancer Cell. 29, 783-803 (2016).

29. Hong, A. W., Meng, Z. \& Guan, K. L. The Hippo pathway in intestinal regeneration and disease. Nat. Rev. Gastroenterol. Hepatol. 13, 324-337 (2016).

30. Ramos, Azucena \& Fernando, D. Camargo. The Hippo signaling pathway and stem cell biology. Trends Cell Biol. 22, 339-346 (2012).
31. Pan, D. The hippo signaling pathway in development and cancer. Dev. Cell. 19, 491-505 (2010).

32. Camargo, F. D. YAP1 increases organ size and expands undifferentiated progenitor cells. Curr. Biol. 17, 2054-2060 (2007).

33. Shao, D. D. et al. KRAS and YAP1 converge to regulate EMT and tumor survival. Cell 158, 171-184 (2014)

34. Oh. et al. Liver regeneration requires Yap1-TGF $\beta$-dependent epithelialmesenchymal transition in hepatocytes. J. Hepatol. 69, 2 (2018).

35. Li, N. et al. Helicobacter pylori CagA promotes epithelial mesenchymal transition in gastric carcinogenesis via triggering oncogenic YAP pathway. J. Exp. Clin. Cancer Res. 37, 280 (2018).

36. Sahin $U$. et al. First-in-human phase $\mid / \|$ dose-escalation study of IMAB027 in patients with recurrent advanced ovarian cancer (OVAR): Preliminary data of phase I part. Int. J. Organic Evolution. https://doi.org/ 10.1111/evo.12140 (2015). 